\title{
An Unusual Case of Primary Peritoneal Mesothelioma
}

\author{
D Belekar, A Desai, V Dewoolkar, J Anam, M Parab
}

\section{Citation}

D Belekar, A Desai, V Dewoolkar, J Anam, M Parab. An Unusual Case of Primary Peritoneal Mesothelioma. The Internet Journal of Surgery. 2008 Volume 20 Number 1.

\begin{abstract}
Peritoneal mesothelioma is a rare disease, but it is increasing in frequency. Because of its unusual nature, the disease has not been clearly defined either in terms of its natural history, diagnosis, or management. Peritoneal mesothelioma patients generally present with two types of symptoms and signs; those with abdominal pain, usually localized and related to a dominant tumor mass with little or no ascites and those without abdominal pain, but with ascites and abdominal distention. Prognosis as determined by clinical presentation, the completeness of cytoreduction, and gender (females survive longer than males) appears to be improved by the use of intraperitoneal chemotherapy. We would like to report a case where our patient primarily presented with peritoneal mesothelioma without pleural involvement that responded well to standard wide surgical excision and postoperative chemotherapy.
\end{abstract}

\section{CASE HISTORY}

A 60-year-old Hindu housewife, hailing from Chennai, came with chief complaints of a swelling over the anterior abdominal wall, slightly right of the midline for 2 months. The patient had pain in the abdomen associated with the swelling. She also had persistent cough without any expectoration, low-grade fever and breathlessness for the last 6 months. She noticed the swelling in the abdominal wall, which was of the size of a small lemon. It progressively increased to twice its dimensions since then. Pain was dull aching in nature and was not associated with food intake. It was not radiating, either. The patient took private treatment for one month but it did not relieve her of her complaints and she finally came to our institute for further management.

The patient was a known diabetic and was taking injection insulin at home regularly. Her diabetes was under control at the time of admission. She had no other major illness or any surgery done in the past. She had no addictions.

She was investigated at Chennai ( 2 months back). Her chest $\mathrm{x}$-ray showed a left perihilar opacity extending up to the left hilum and an elevated dome of diaphragm. FNAC showed a poorly differentiated malignant tumor, not lymphoma. Bronchoscopy was also done which showed left main bronchus inflammation. Broncho-alveolar lavage (BAL) showed only inflammatory cells and no malignancy. 2-D echo showed a LVEF of $74.2 \%$ with trivial pericardial effusion.
On examination, there was a solitary swelling measuring $5 \mathrm{x}$ $5 \times 2 \mathrm{~cm}$ located in left hypochondrium and lumbar area with skin adherent to the swelling and it was shiny with a smooth surface. There were no scars/sinuses or dilated veins over the swelling. It was also fixed to underlying muscles as confirmed by Carnet's test.

\section{Figure 1}

Figure 1: Gross external appearance of the abdominal swelling.

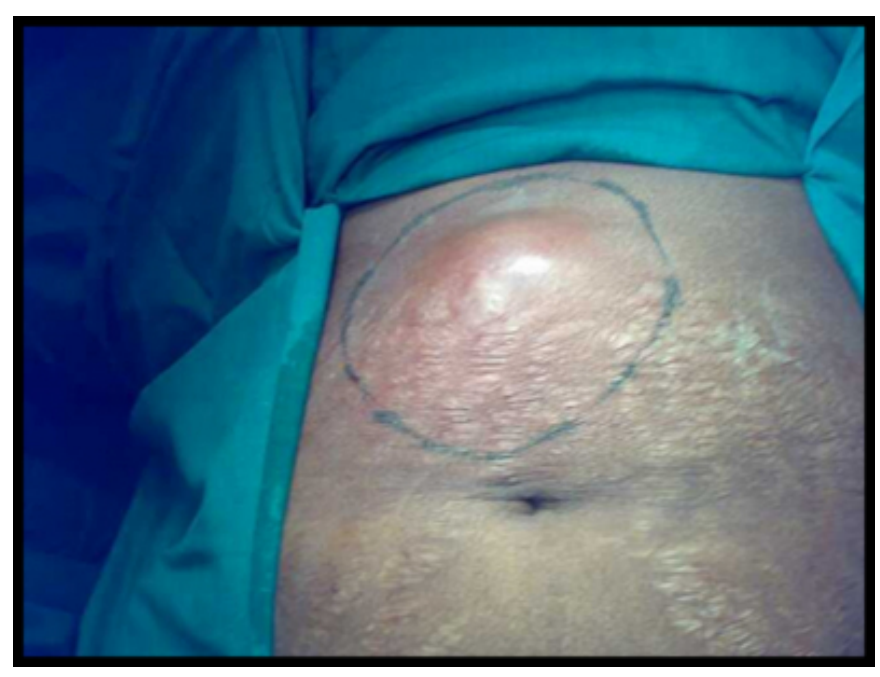

Her systemic examination showed bilateral decreased air entry.

She was investigated again. Her FNAC showed a malignant spindle-cell neoplasm. Her chest X-ray showed a left lower 
zone consolidation and collapse of the left lower lobe. The rest of her reports was within normal limits. A decision to perform wide excision biopsy was taken. She underwent wide excision biopsy keeping $2.5 \mathrm{~cm}$ margins. The tumor was attached to the transverse colon infiltrating the serosa and muscularis. There were adhesions with stomach and small intestine, but they were easily separated with blunt dissection. The liver showed a solitary small nodule. Its excision biopsy was sent along with the resected involved colon. Tension sutures were taken over an abdominal drain The drain was removed after 5 days. Suture removal was done after 15 days.

At pathology, on gross examination, an $11 \times 11 \times 10 \mathrm{~cm}$ specimen involving skin, subcutaneous tissue, tumor, muscles, peritoneum and transverse colon was described. Cut section showed a gray-white tumor measuring $10 \mathrm{x} 9 \mathrm{x}$ $8 \mathrm{~cm}$. It was infiltrating subcutaneous fat and was $0.5 \mathrm{~cm}$ away from the skin.

\section{Figure 2}

Figure 2: Cut section in profile showing all involved layers.

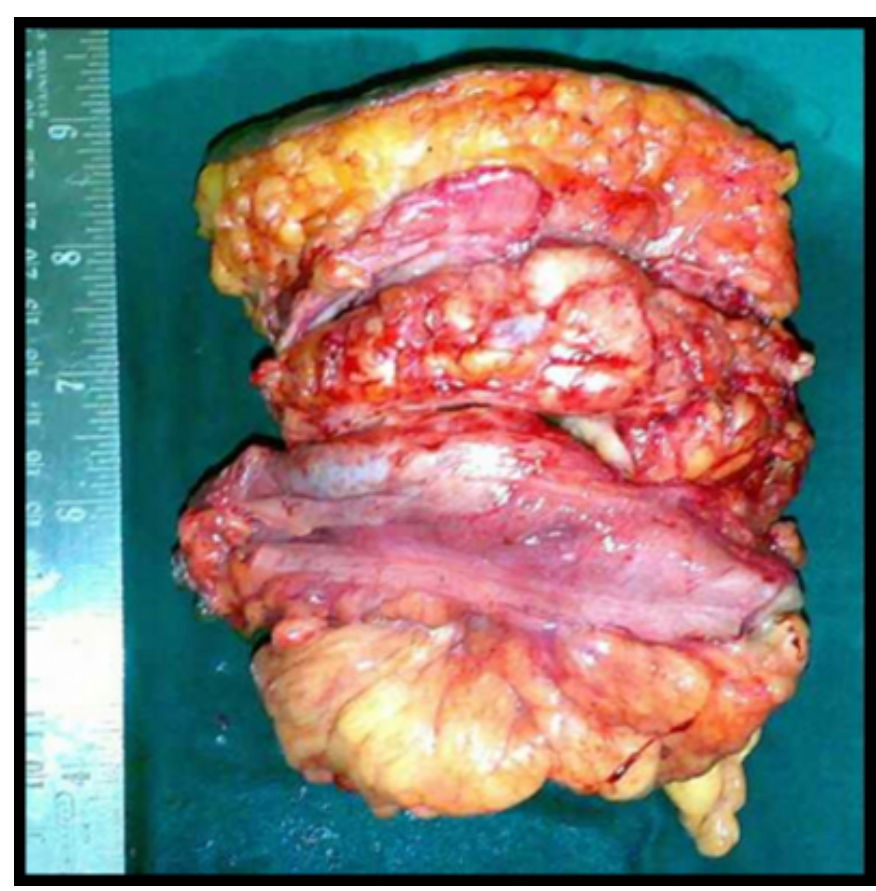

Histopathology showed primary mesothelioma involving the peritoneum with tumor-free margins. Lymphovascular emboli were seen. Liver metastasis was positive. The omentum also showed metastasis. The distal surgical margin was tumor-free. Immunohistochemistry (IHC) was asked to confirm the diagnosis. IHC from Tata Memorial Hospital showed malignant mesothelioma of predominantly epithelial type. Tumor cells expressed CK5/6, CK and EMA, while they were negative for CK-20 and Calretinin.

The patient recovered well post-operatively along with vigorous chest physiotherapy. Her suture removal was done after 15 days. She was offered chemotherapy postoperatively.

\section{DISCUSSION}

Mesothelioma is a neoplasm originating from the mesothelial cells lining the human body cavities.

Mesothelioma may involve the pleura, less frequently the peritoneum, and, rarely, the pericardium and the tunica vaginalis testis. Peritoneal mesothelioma is usually a rapidly fatal peritoneal surface malignancy with a median survival of less than 1 year. It represents about one fifth to one third of all forms of mesothelioma. Asbestos exposure appears to be causative in some cases of peritoneal mesothelioma, but a search for other carcinogens continues $\left(_{1}\right)$.

Clinically, the vague nature of the symptoms and signs, the long-term presence of these symptoms and signs prior to definitive diagnosis, and the young age of many of these patients led to a delay in diagnosis. Laparoscopy was the most common diagnostic test required for definitively diagnosing the wet type of mesothelioma (64\%). Cytology of fluid removed by paracentesis rarely resulted in a definitive diagnosis $\left({ }_{2}\right)$.

The two clinical types of peritoneal mesothelioma, wet or dry-painful type, have profoundly different appearances on CT examination. In the wet type, there is little or no evidence of a solid tumor. Occasionally, small nodules lining the parietal peritoneal surfaces are evident, especially beneath the right hemidiaphragm. The $\mathrm{CT} /$ radiologic presentation of the dry-painful type of peritoneal mesothelioma may disclose several mass lesions, but often there is a dominant mass isolated to one part of the abdomen. Likewise, the tumor mass is commonly associated with the greater omentum. Usually the symptomatic mass lesion seen on $\mathrm{CT}$ is thought to be an intra-abdominal abscess or a large primary adenocarcinoma. Only at laparotomy is the definitive diagnosis evident in these patients presenting with solid tumor in the absence of ascites $\left(_{3}\right)$.

The preoperative CT is of great value in predicting the benefits of cytoreductive surgery plus intraperitoneal chemotherapy. The CT often predicts complete removal of all visible tumor using peritonectomy procedures $\left(_{4}\right)$. To facilitate successful surgery, the small bowel and its mesentery should be compartmentalized apart from the 
malignancy and there should be no tumor nodules directly adjacent to the small bowel. No disruption of intestinal function like segmental bowel obstruction should be present.

One of the greatest dilemmas encountered in the management of patients with peritoneal mesothelioma involves prior and repeated debulking procedures. In this case, the malignant cells become uniformly distributed over the peritoneal surfaces including small bowel and small bowel mesentery. This leads to solid tumor diffusely distributed in large volumes and intimately attached to the small bowel. In this situation, the patient becomes untreatable by loco-regional treatment strategies.

Occasionally, aggressive systemic chemotherapy results in a transient response, but no long-term survival occurs once fixation of the bowel has taken place.

Approximately $10 \%$ of patients with primary colon cancer have peritoneal carcinomatosis. Up to $30 \%$ of patients with gastric cancer and pancreas cancer have peritoneal seeding at the time of exploration for resection of the primary malignancy. A majority of patients with papillary serous ovarian cancer have peritoneal seeding. The simultaneous occurrence of one of these common cancers coincidentally with a primary peritoneal mesothelioma can occur.

The histopathologic examination by hematoxylin and eosin stain remains an important part of the diagnosis of peritoneal mesothelioma. Cords of cuboidal and polygonal epithelial cells within a mucinous matrix are prominent. A papillary growth pattern that emerges within areas of apparent mesothelial hyperplasia is often evident. Peritoneal mesothelioma represents $20 \%$ to $37 \%$ of all mesotheliomas because of its rarity and the small size of published series, the best estimate of annual incidence in the United States is 200 to 400 new cases.

In a review of the literature, no dominant therapeutic guidelines for peritoneal mesothelioma are found. Most papers published are clinicopathological retrospective reviews or case reports compiling disparate therapeutic experiences, including the use of systemic chemotherapy, whole abdominal radiation, and intraperitoneal treatments $\left(_{5}\right)$ with compounds such as colloidal radioactive "P and 19" Au, thiotepa, and bleomycin. The positive predictive factors for survival identified by univariate analysis included: (a) female gender, (b) good health status, (c) presentation in the absence of increased abdominal girth, (d) low peritoneal cancer index, (e) complete cytoreduction, and (f) re-do surgery (for selected patients) $\left.{ }_{6}\right)$.

There is no statistical indication that intraperitoneal chemotherapy resulted in survival benefit. With the current state of knowledge, a recommendation for intraperitoneal cisplatin, doxorubicin, and paclitaxel as a treatment for peritoneal mesothelioma, combined with cytoreductive surgery, may be helpful ${ }_{7}$ ); however, large multicenteric, double-blind randomized controlled trials regarding this entity should be carried out to achieve standardization.

Our patient has undergone wide local excision involving all the layers. Post-operatively, she was offered systemic chemotherapy to which she responded quite well. She regained her appetite and was healthy at follow-up without any evidence of recurrence.

\section{References}

1. Averbach AM, Sugarbaker PH. Peritoneal mesothelioma: Treatment approach based on natural history. Cancer Treat Res 1996; 81: 193-211.

2. Moertel C. Peritoneal mesothelioma. Gastroenterology

1972; 63: 346-50.

3. Plaus WJ. Peritoneal mesothelioma. Arch Surg 1988; 123 : 763-6.

4. Jacquet P, Jelinek JS, Sugarbaker PH: Abdominal computed tomographic scan in the selection of patients with mucinous peritoneal carcinomatosis for cytoreductive surgery. J Am Coll Surg 1995; 181: 530-538.

5. Ma GY, Bartlett DL, Reed E, et al. Continuous hyperthermic peritoneal perfusion with Cisplatin for the treatment of peritoneal mesothelioma. Cancer J Sci Am 1997; 3: 174-9.

6. Antman K, Osteen R, Klegar K, et al. Early peritoneal mesothelioma: A treatable malignancy. Lancet 1985; 2: 977-81.

7. Park BJ, Alexander HR, Libutti SK, et al: Treatment of primary peritoneal mesothelioma by continuous hyperthermic peritoneal perfusion (CHPP). Ann Surg Oncol 1999; 6: 582-590. 


\section{Author Information}

Dnyanesh M. Belekar, MBBS, MS, FCPS, DNB, MNAMS, FMAS, FAIS.

Associate Professor, Dept. of General Surgery, K. J. Somaiya Medical College

Amit A. Desai, MBBS, MS, DNB.

Senior Post Graduate Registrar, Dept. of General Surgery, K. J. Somaiya Medical College

Vinayak V. Dewoolkar, MBBS, MS

Dean and Professor of Surgery, Dept. of General Surgery, K. J. Somaiya Medical College

Jay R. Anam, MBBS

Senior Registrar, Dept. of General Surgery, K. J. Somaiya Medical College

Mrunal B. Parab, MBBS

Surgery Resident, Dept. of General Surgery, K. J. Somaiya Medical College 